\title{
The somite-secreted factor Maeg promotes zebrafish embryonic angiogenesis
}

\author{
Xin Wang ${ }^{1, *}$, Wei Yuan ${ }^{1, *}$, Xueqian Wang ${ }^{1, *}$, Jialing $Q i^{2}$, Yinyin Qin ${ }^{1}$, Yunwei Shi ${ }^{1}$, Jie \\ Zhang ${ }^{2}$, Jie Gong ${ }^{3}$, Zhangji Dong ${ }^{1}$, Xiaoyu Liu ${ }^{1}$, Chen Sun ${ }^{1}$, Renjie Chai ${ }^{1,4}$, Ferdinand \\ Le Noble ${ }^{5}$, Dong Liu ${ }^{1}$ \\ ${ }^{1}$ Co-innovation Center of Neuroregeneration, Jiangsu Key Laboratory of Neuroregeneration, Nantong University, Nantong, \\ China \\ ${ }^{2}$ Medical College, Nantong University, Nantong, China \\ ${ }^{3}$ School of life science, Nantong University, Nantong, China \\ ${ }^{4}$ Key Laboratory for Developmental Genes and Human Disease, Ministry of Education, Institute of Life Sciences, Southeast \\ University, Nanjing, China \\ ${ }^{5}$ Department of Cell and Developmental Biology, Karlsruhe Institute of Technology, Karlsruhe, Germany \\ *These authors have contributed equally to this work \\ Correspondence to: Dong Liu, email: liudongtom@gmail.com, tom@ntu.edu.cn \\ Keywords: Maeg, angiogenesis, integrin, Notch, zebrafish \\ Received: May 09, $2016 \quad$ Accepted: October 12, $2016 \quad$ Published: October 21, 2016
}

\section{ABSTRACT}

MAM and EGF containing gene (MAEG), also called Epidermal Growth Factor-like domain multiple 6 (EGFL6), belongs to the epidermal growth factor repeat superfamily. The role of Maeg in zebrafish angiogenesis remains unclear. It was demonstrated that maeg was dynamically expressed in zebrafish developing somite during a time window encompassing many key steps in embryonic angiogenesis. Maeg loss-offunction embryos showed reduced endothelial cell number and filopodia extensions of intersegmental vessels (ISVs). Maeg gain-of-function induced ectopic sprouting evolving into a hyperbranched and functional perfused vasculature. Mechanistically we demonstrate that Maeg promotes angiogenesis dependent on RGD domain and stimulates activation of Akt and Erk signaling in vivo. Loss of Maeg or Itgb1, augmented expression of Notch receptors, and inhibiting Notch signaling or DII4 partially rescued angiogenic phenotypes suggesting that Notch acts downstream of Itgb1. We conclude that Maeg acts as a positive regulator of angiogenic cell behavior and formation of functional vessels.

\section{INTRODUCTION}

A number of secreted factors produced by surrounding cells and tissues regulate angiogenesis through mediating endothelial cells (ECs) differentiation, proliferation, and migration [1-3]. The epidermal growth factor (EGF) repeats is a widely distributed module in many different proteins in single or multiple copies that was first described in 1972 within the EGF protein $[4,5]$. Interestingly, EGF-like proteins such as Betacellulin (BTC) [6], Heparin-binding EGF-like growth factor (HBEGF) [7-10], and EGFL7 [11-17] have been demonstrated to play vital roles in angiogenesis and endothelial cell behaviors. MAEG (MAM and EGF containing gene), also named EGFL6, was first identified in 1999 as an EGF repeat-containing protein [18]. Adipose secreted MAEG has been proved to promote proliferation of adipose tissue-derived stromal vascular cells [19]. Moreover, that osteoblastic-like cells express MAEG that is capable of promoting endothelial cell migration and angiogenesis via ERK activation [20]. However, the functional analysis of Maeg involved in embryonic vascular development of vertebrate in vivo is thus far lacking.

The vascular network develops in a conserved manner in all vertebrates. The zebrafish (Danio rerio) model system offers distinct advantages for in vivo studies of the vascular development [21]. In the present study, we analyzed the expression dynamics of Maeg in zebrafish developing somite by using whole-mount in situ hybridization, and immunostaining. Then we examined 
whether zebrafish maeg regulates angiogenesis in vivo through loss- and gain-of-function analysis. Further more, we investigated the potential mechanism underlying maeg regulating zebrafish embryonic angiogenesis.

\section{RESULTS}

\section{Maeg dynamically expressed in zebrafish developing somite}

To investigate the expression dynamics of Maeg in zebrafish developing somite, we did the detailed whole amount in situ hybridization (WISH) analysis using digoxigenin-labeled antisense probes. Consistent with previous report [22], the hybridization signal was apparently shown in myotome at $12 \mathrm{hpf}$ and increasingly maintained at $15 \mathrm{hpf}$ (Figure 1A-1E). The somital expression of maeg reached its peak at around 22 26 hpf and decreased hereafter (Figure 1F-1G"'). The hybridization signal of maeg in somite became very weak at $30 \mathrm{hpf}$ and barely detectable at 48 and $60 \mathrm{hpf}$ (Figure 1H-1J). Taken together, maeg dynamically expressed in zebrafish developing somite during a time window encompassing many key steps in embryonic angiogenesis. In addition, the expression dynamics of maeg in hindbrain was similar with that in somites (Figure 1A-1J). Whole-mount immunostaining analysis of Maeg in Wild type (WT) embryos showed that Maeg protein was present in somites and highly accumulated in somite borders (Figure 1K).

\section{Establishment of maeg knockout mutant line by TALEN}

To do the loss of function analysis on maeg gene in zebrafish, TALEN was utilized to establish maeg knockout mutant line. To ensure complete disruption of functional proteins, we chose the TALEN target sites near the translation start codon in the first exon of zebrafish maeg (Figure 2A, Supplementary Figure S1A). Then we built two constructs containing left and right arms of maeg TALEN respectively. The in vitro synthesized mRNAs of the two arms were microinjected into the cytoplasm of 1-cell stage zebrafish embryos. TALEN in vivo activity was analyzed by HRMA. The TALEN proteins turned out to efficiently induce mutations in the targeting site (Supplementary Figure S1B). To evaluate the mutagenesis frequency, a 150-bp genomic DNA fragment containing the target site was PCR amplified from 32 TALEN injected embryos at $24 \mathrm{hpf}$ and sequenced. Sequence analysis revealed that the mutated rate of maeg alleles was $43.8 \%$ (14/32) and 8 types of mutations (Figure 2B). The remaining siblings of these F0 embryos were raised to adulthood. The healthy F0 founders carrying somatic mutations were out-crossed with wild type fish to obtain F1 offspring. Among the adult F1 offspring, 3 types of mutations were identified via PCR amplification and sequencing with fin-clipped genomic
DNAs (Figure 2C, S1C). The mutated alleles included an 8-bp deletion, a 7-bp deletion and a 1-bp insertion, which all result in reading frame shift and premature translation termination (Figure 2C). The Maeg protein ablation in 7-bp deletion line was confirmed by western blot analysis (Supplementary Figure S2C). For the subsequent experiments, the 7-bp deletion mutant line was selected to outcross with $\mathrm{Tg}$ (flila:nEGFP) and $\mathrm{Tg}(\mathrm{kdrl}: E G F P)$ transgenic lines for studying angiogenesis. Homozygous maeg mutant individuals $\left(\mathrm{maeg}^{-/}\right)$were obtained in F3 offspring.

\section{Loss of Maeg impairs intersegmental vessels branching angiogenesis}

To investigate the role of Maeg in embryonic angiogenesis, we examined vascular development of maeg $^{-/-} \mathrm{Tg}(\mathrm{kdrl}: E G F P)$ zebrafish embryo at different stages using in vivo confocal imaging. In the maeg mutants, although the position of the initial sprout formation was not affected (Figure 3A, S2B), at $24 \mathrm{hpf}$ the length of intersegmental vessel (ISV) is significantly shorter than that of control (Figure 3B, S2B). At $30 \mathrm{hpf}$ stage the ISVs grew only halfway through their ventral trajectory and usually failed to cross the horizontal myoseptum (Figure 3A, 3B). As a consequence, formation of the dorsal lateral anastomotic vessel (DLAV) was severely disturbed (Figure 3A). Loss of maeg did not apparently affect the diameter of axial vessels, including DA and posterior cardinal vein (PCV), which are formed via vasculogenesis (Figure $3 \mathrm{C}$ ). To confirm the angiogenic phenotype of maeg mutants, we knocked down maeg using two mopholinos (MO, a mopholino for blocking translation and a morpholino for modifying pre-mRNA splicing), whose specificity were validated by Western Blot and RT-PCR analysis as previous reported [22]. Confocal imaging analysis revealed that morpholinomediated knockdown of maeg phenocopied the angiogenic defects of maeg mutants (Figure 3A, 3B, 3C).

Due to the localization of Maeg in somites and the borders, we evaluated weather the angiogenesis defects in maeg mutants were caused by the abnormalities of somites and the borders. Through the phalloidin staining, immunostaining and microscope imaging analysis, we did not observe the obvious defects of somites and the borders in maeg deficiency embryos (Figure 3A, S3A-S3D). We also examined weather maeg is involved in embryonic hindbrain development in $\operatorname{Tg}(h u C: E G F P)$ embryos. Confocal imaging analysis of the fluorescence labeled neurons in $\operatorname{Tg}(h u C: E G F P)$ embryos revealed normal hindbrain development in maeg loss of function zebrafish (Supplementary Figure S3E, S3F).

\section{Maeg overexpression causes excessive branching}

To further determine the role of maeg in angiogenesis, we did the maeg gain of function analysis in zebrafish 
embryos. We observed the excessive branching of ISVs in maeg mRNA injected embryos (Figure 4A). In control 48 hpf embryos, the paralleled ISVs from DA or PCV are separated single tubes and directly connected to the DLAV (Figure 4A-4i). In maeg up-regulated embryos, there were usually two sorts of phenotypes of excessively branched
ISV. One of those is ISV from the axial vessels gave rise to two sprouts and connected with DLAV respectively, forming Y-shaped structure (Figure 4A-4ii). And another is that one of the ISVs branched an additional sprout from its middle position to connect with adjacent one, forming H-shaped structure (Figure 4A-4ii; Supplementary Figure S4A). More
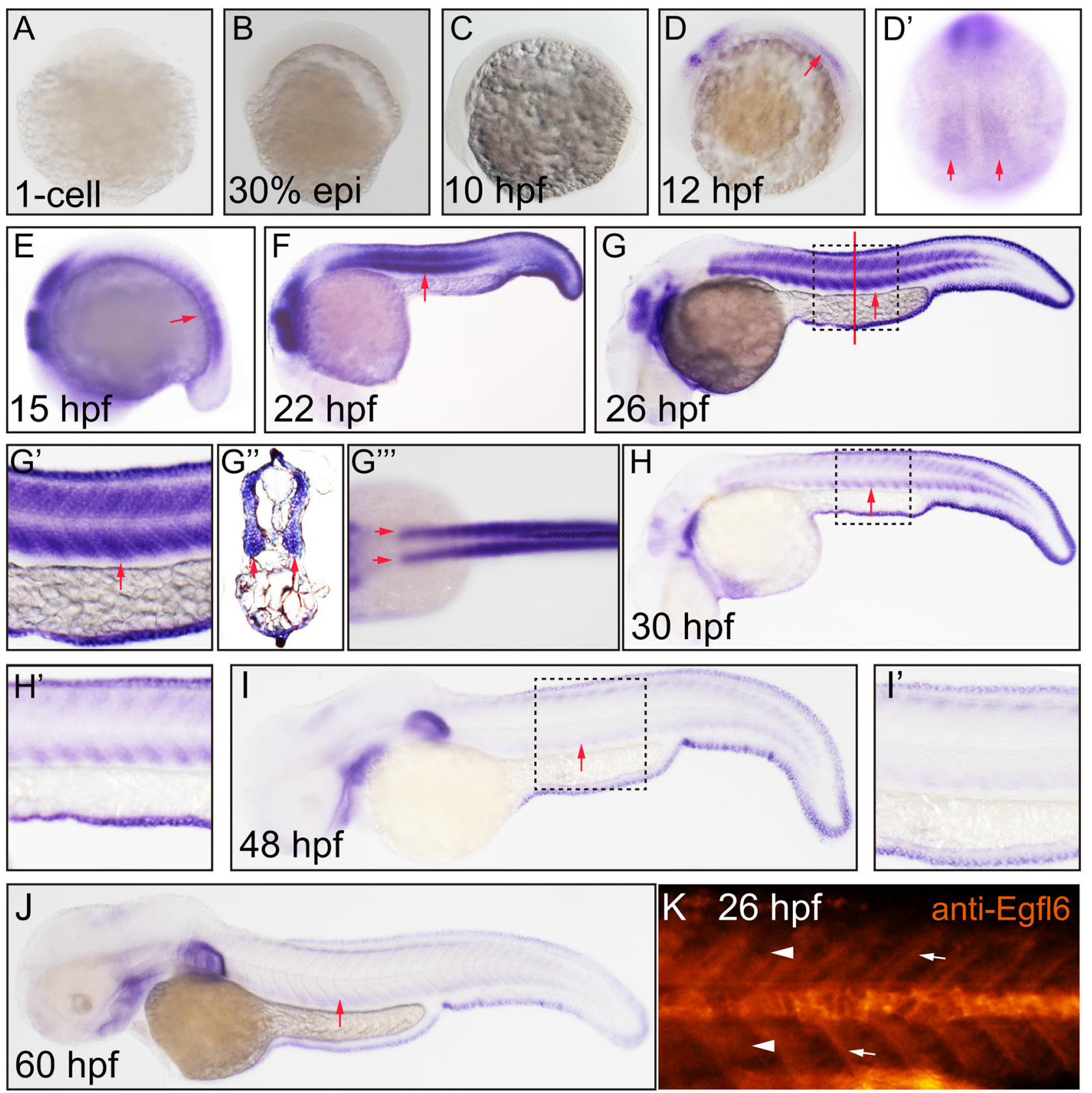

Figure 1: Maeg dynamically expressed in zebrafish developing somite. Expression of maeg was analyzed by whole mount in situ hybridization and whole mount antibody staining. A. 1-cell, lateral view, no staining. B. 30\% epiboly, lateral view, no staining. C. 10 hpf, lateral view, no staining. D. 12 hpf, lateral view, arrow indicates somite. D'. 12 hpf, lateral view, arrows indicate somite. E. 15 hpf, lateral view, arrow indicates somite. F. 22 hpf, lateral view, arrow indicates somite. G. $26 \mathrm{hpf}$, lateral view, arrow indicates somite, square in dash line indicates the magnified region in G'. red line indicates the section position G', G'". 26 hpf, dorsal view, arrows indicate myotomes. H. $30 \mathrm{hpf}$, lateral view, arrow indicates somite, square in dash line indicates the magnified region in H', I. 48 hpf, lateral view, arrow indicates somite, square in dash line indicates the magnified region in I', J. $60 \mathrm{hpf}$, lateral view, arrow indicates somite. K. 26 hpf, lateral view, arrowheads indicate somites, arrows indicate somite borders. 
than $80 \%$ maeg over expression embryos had no less than one excessively branched ISV (Figure 4B). We also noted these aberrant branching points mostly happened in the dorsal half of the ISVs. In some cases, these phenotypes combined or repeated to form more complex structures (Figure 4A-4ii, Supplementary Figure S4B, S4C). These aberrant vessel connections formed a lumen and perfused with blood flow (Figure 4A). The diameter of ISV in maeg gain of function embryos is slightly larger than that in control embryos (Figure 4C). And over expression of maeg did not significantly affect the diameters of axial vessels (Figure 4D, 4E). Furthermore we checked the identity of those hyperbranched ISVs and found around $75 \%$ of them were arteries. In addition we observed significant

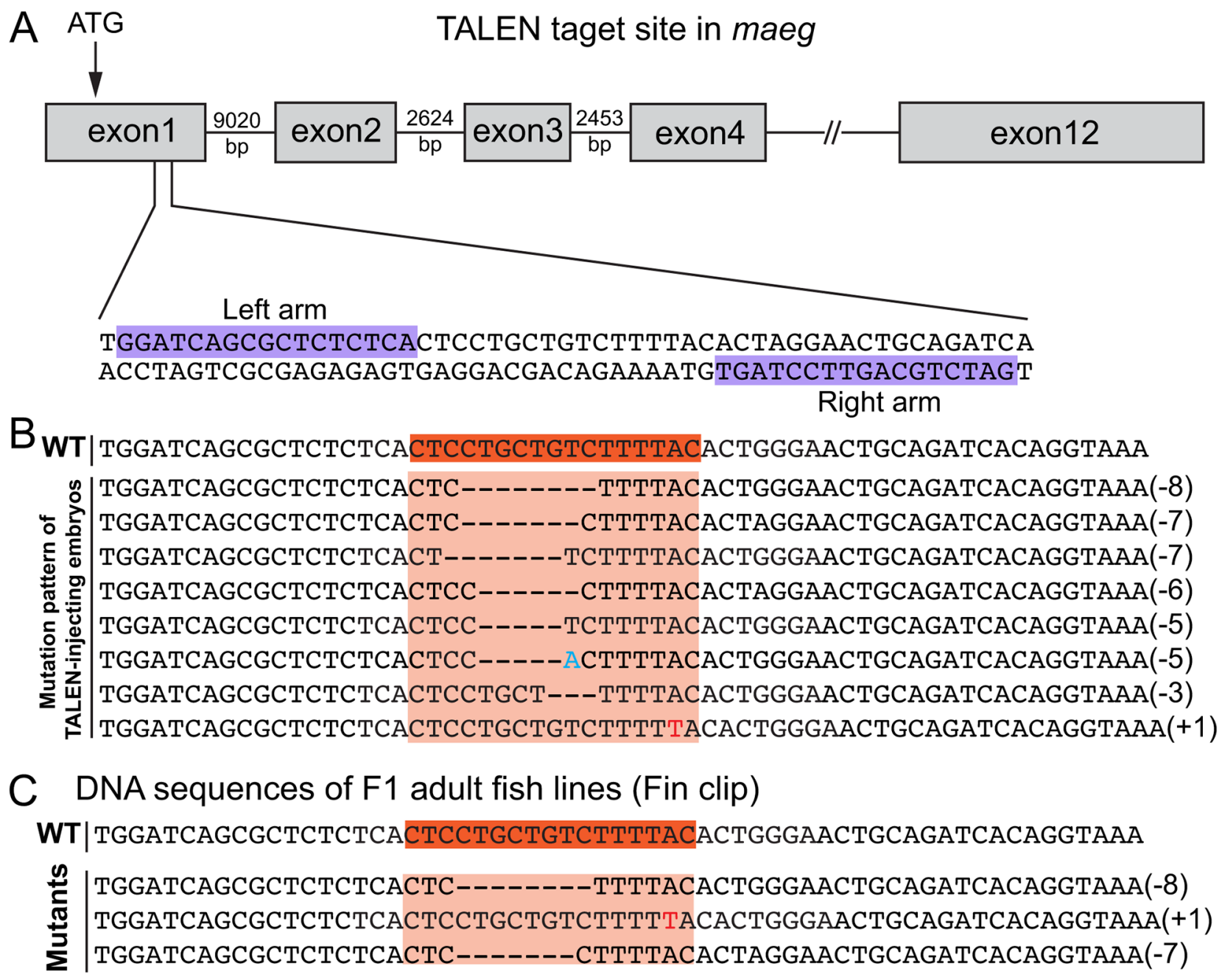

\section{Protein sequences of $F 1$ adult fish lines}

WT (506AA)

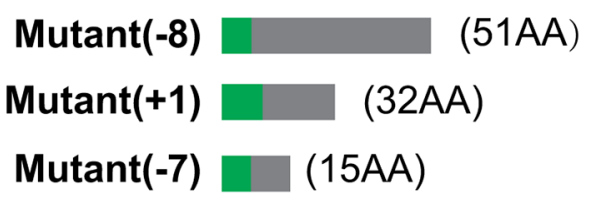

Figure 2: Generation of zebrafish maeg mutant using TALENs. A. Schematic diagram showing TALEN targeting site on the first exon of maeg gene. Starting codon (ATG) site is indicated by arrow. The left and right TALEN targeting sites are highlighted in purple. B. Mutation pattern of TALEN-injecting embryos. Numbers in the brackets show the number of nucleotides were deleted $(-)$ or inserted $(+)$. Inserted nucleotide is in red. WT, wild-type. C. Three heritable mutants were identified by screening. F0 founder fish were out-crossed with WT fish to produce F1, and the DNA extracted from tail fins of F1 adults were used for identification of heritable mutants by sequencing. D. Schematic diagram showing the predicted proteins encoded by the three mutated alleles. The mutants are reading frameshift mutations that result in truncated proteins. The gray rectangles indicate the wrong coded amino acid sequences. 
hyperbranching in subintestinal vessels (SIV) (Figure 4F). Overexpression of maeg resulted in the SIV became a much more complex structure, with the ECs numbers and branch points increased dramatically (Figure 4F, 4G, 4H). Interestingly, knot-like structures were observed in some of the ISVs in maeg gain of function embryos (Figure 4I). Around 28\%(5 of 18) of the maeg over expression embryos we analyzed exhibited this sort of phenotype. However, we did not find this structure in control embryos. The knot-like structure was also shown to continue branching and form more complex structures (Figure 4I-4iii, -vi).

\section{Maeg regulates the endothelial tip cell behaviors}

Since maeg was required for ISVs outgrowth, we reasoned that it might play a role in governing endothelial tip cell behaviors. To determine if this was the case and study the cellular mechanisms underlying the branching angiogenic defect of ISVs caused by loss of maeg, we analyzed the effects of maeg on tip cell behaviors in zebrafish embryos. Firstly we examined the tip cell proliferation, and migration using in vivo time-lapse imaging of $\operatorname{Tg}($ flila:nEGFP)
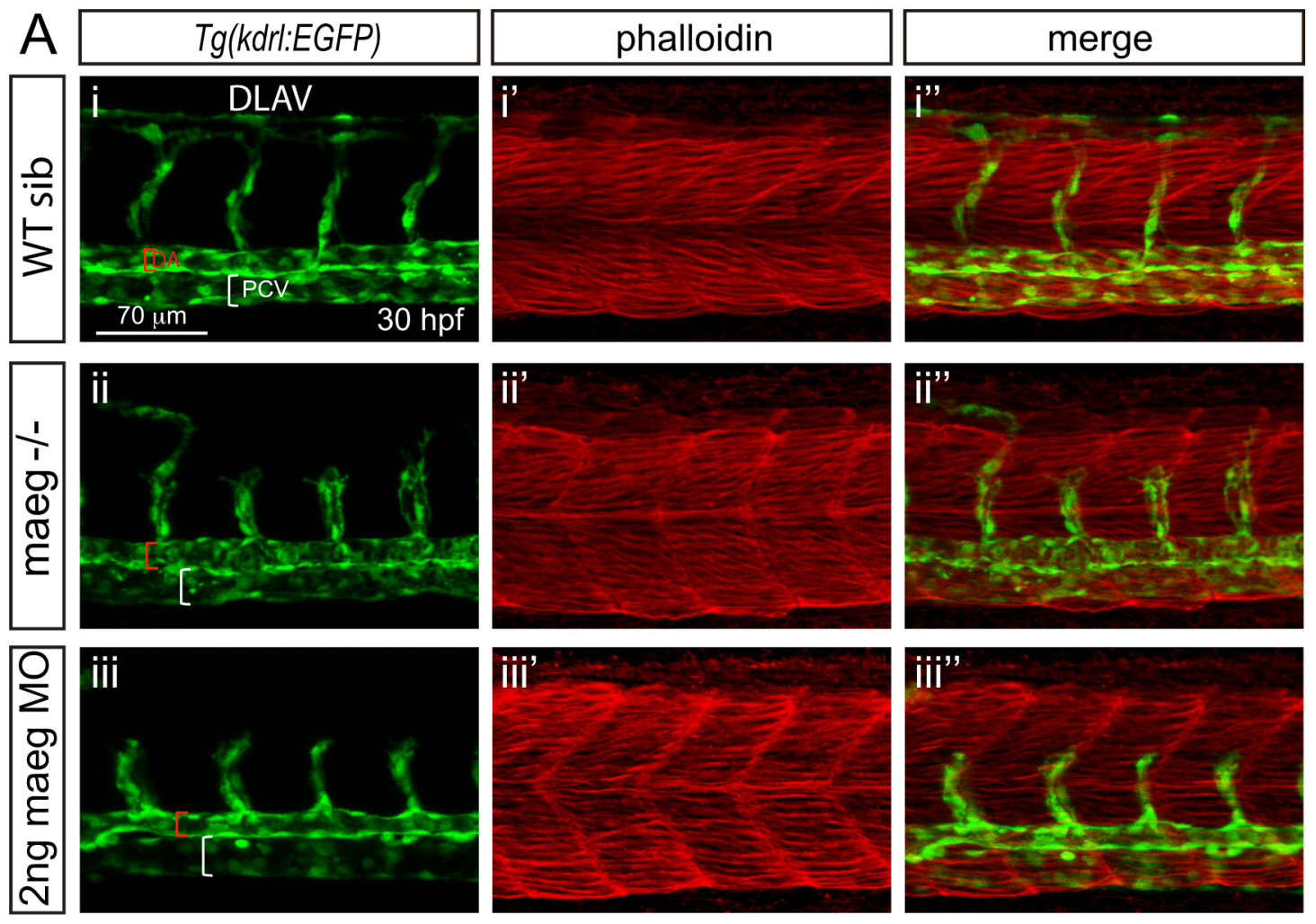

$\mathrm{B}$

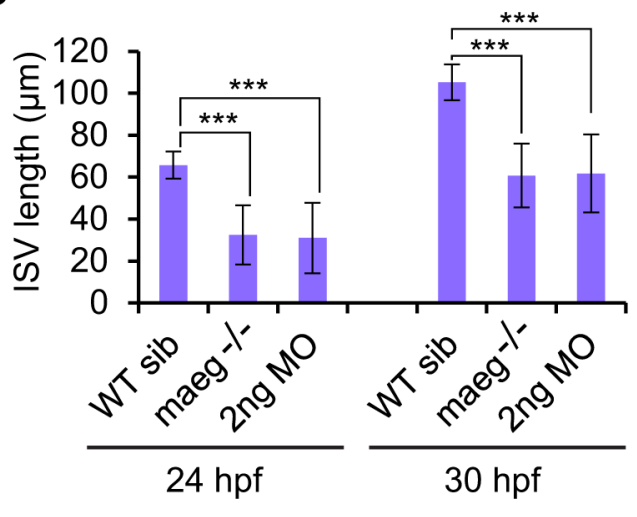

C

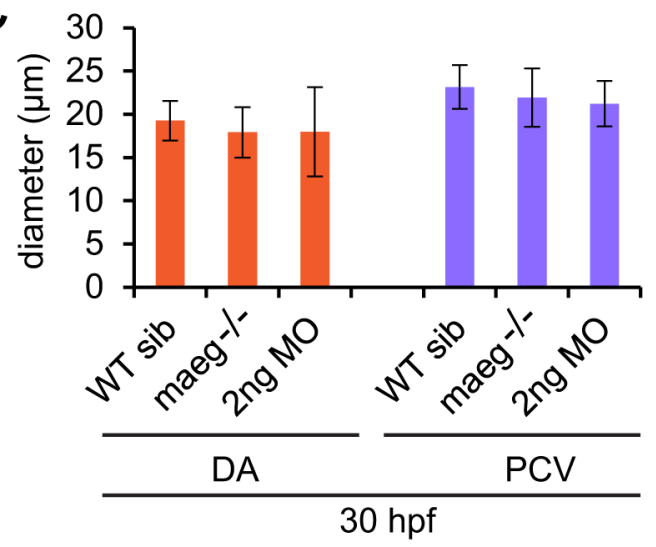

Figure 3: maeg loss of function results in the blood vessel morphogenesis defects in zebrafish embryos. A. Confocal imaging analysis of trunk vascular and somital morphology in WT, maeg ${ }^{-/-}$and maeg morphants $\operatorname{Tg}(\mathrm{kdrl}: E G F P)$ embryos at 30 hpf. Red and white square brackets indicate the lumen of the DA and PCV, respectively. DA, dorsal aorta; PCV, posterior cardinal vein; and DLAV, dorsal longitudinal anastomotic vessel. B. The statistics of ISV length in WT, maeg ${ }^{-/}$and maeg morphants. One-Way ANOVA; $* * *, P<0.001$. C. The statistics of DA and PCV lumen size at $30 \mathrm{hpf}$. Error bars indicate stdev. 
embryos, in which EGFP accumulates in nucleus in ECs [23]. In control embryos, the endothelial tip cells of ISV initiated from DA at around $20 \mathrm{hpf}$ and migrated towards the dorsal direction along their trajectory, as described in previous work [16]. Once arriving at the horizontal myoseptum, tip cells in most ISVs underwent proliferation into 2 ECS (Figure 5A), and one of the daughter cell continuously migrate to the dorsal roof to form DLAV (Figure 5A). In the absence of maeg, the ISV tip cells migrated from DA later than the stage of control (Figure 5A). Furthermore, the subsequent migration of tip cells from the horizontal myoseptum to the DLAV was slower than that in the control embryos (Figure 5C). Some of the tip cells still stopped at the horizontal myoseptum in $33 \mathrm{hpf}$ maeg mutants (Figure $5 \mathrm{~A})$. In addition, half of the tip cells failed to proliferate
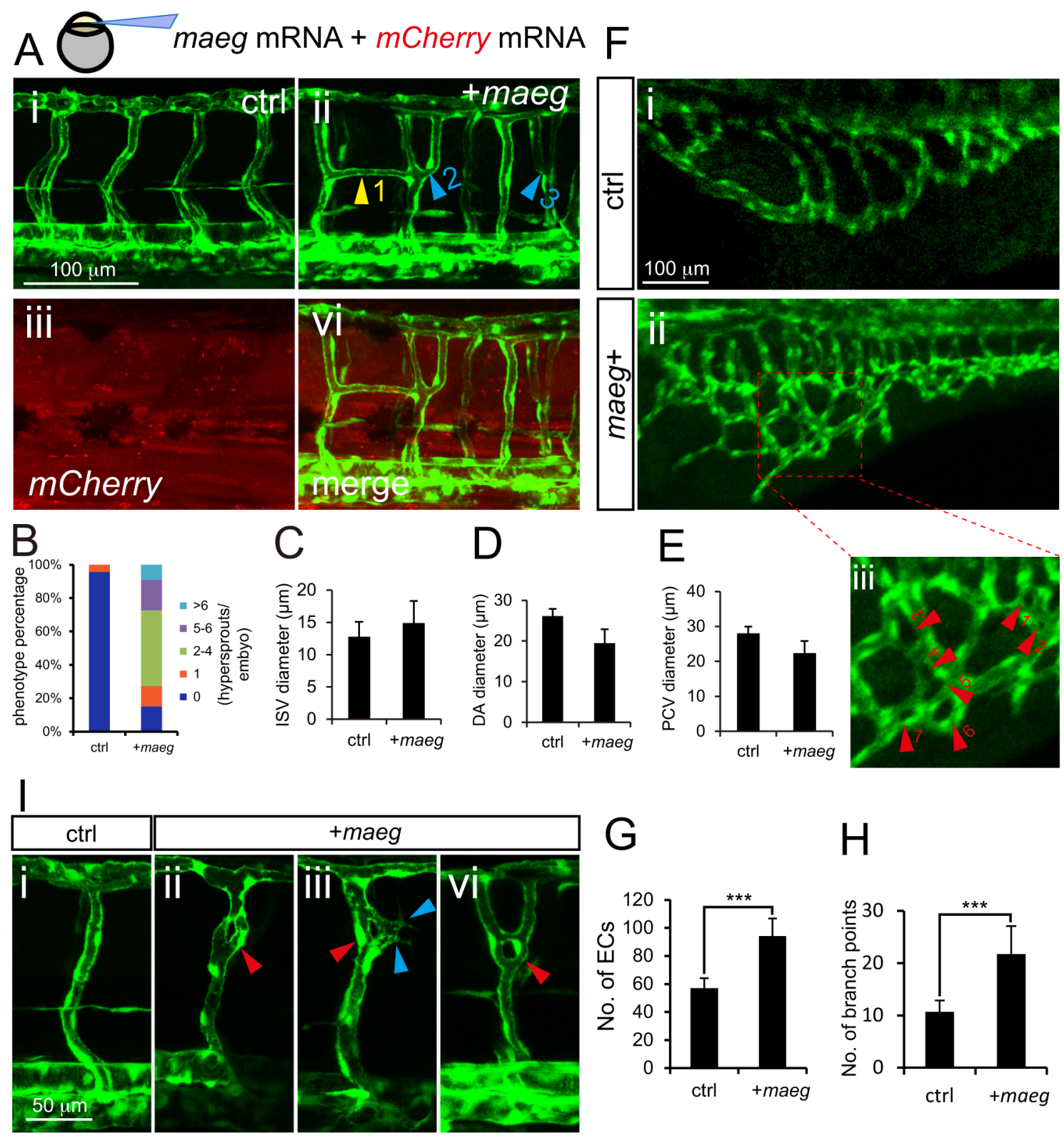

Figure 4: Maeg overexpression causes excessive branching. A. Confocal imaging analysis of trunk vascular morphology in control and maeg/mCherry mRNA mixture injected $T g(k d r l: E G F P)$ embryos at 48 hpf. Yellow arrowhead indicates the aberrant vessel connected two adjacent ISVs. Blue arrowheads indicate Y-shaped ISVs. B. The statistics of hyperbranching sprouts in maeg up-regulated embryos. C-E. The statistics of ISV, DA and PCV lumen size at 48 hpf. Error bars indicate s.e.m. F. Morphology of subintestinal vessel (SIVs) in 72 hpf $T g$ (flila:EGFP) embryos injected with control mRNA or maeg mRNA. G. The statistics of branch point number. Student's t-test; $* * *, P<0.001$. H. Quantification of ECs nuclei number in SIVs. Student's t-test; $* * *, P<0.001$. I. Confocal imaging analysis of ISVs morphology in control and maeg mRNA injected $T g(k d r l: E G F P)$ embryos at 48 hpf. Red arrowheads indicate knot-like structures. Blue arrowheads indicate angiogenic sprouts. 
A $23 \mathrm{hpf} \operatorname{Tg}($ fli1a:nEGFP)
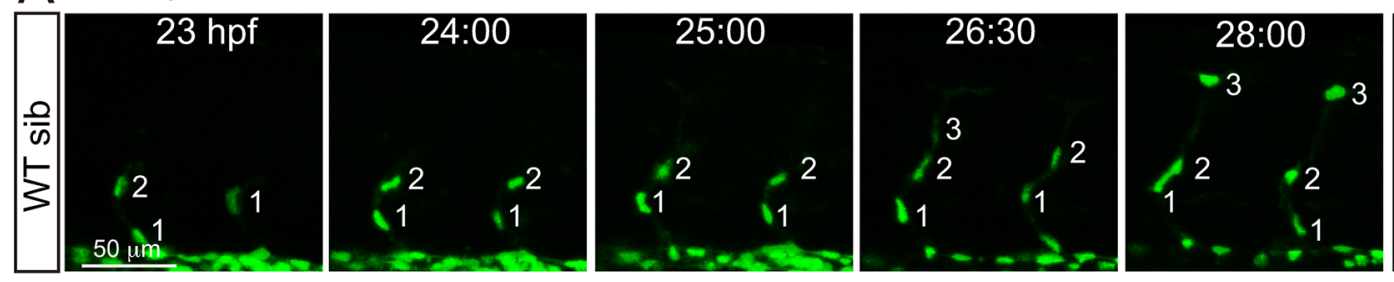

$29 / 33 \mathrm{hpf}$
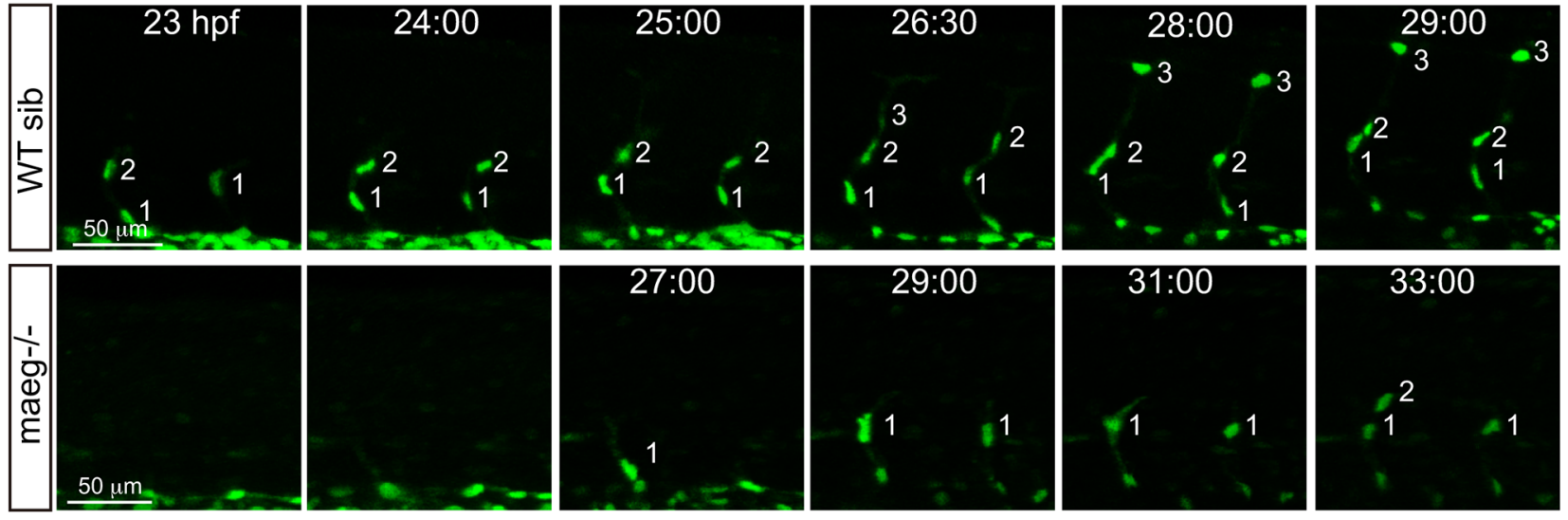

B
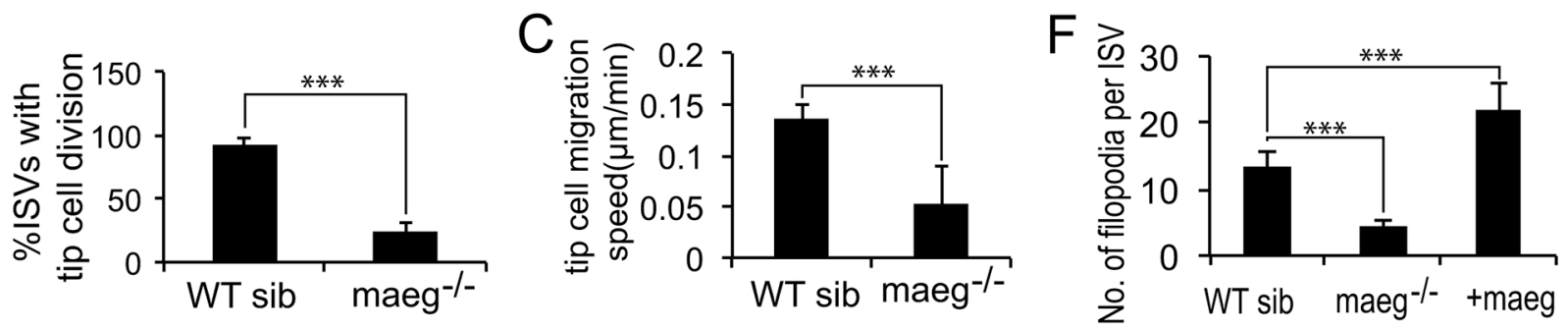

D $24 / 27 \mathrm{hpf}$
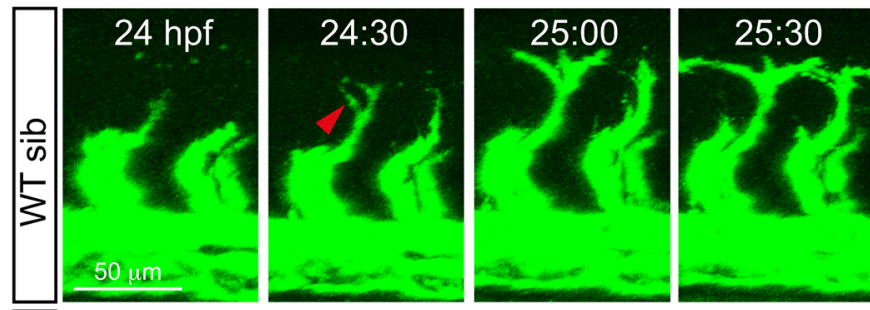

26:30/30 hpf
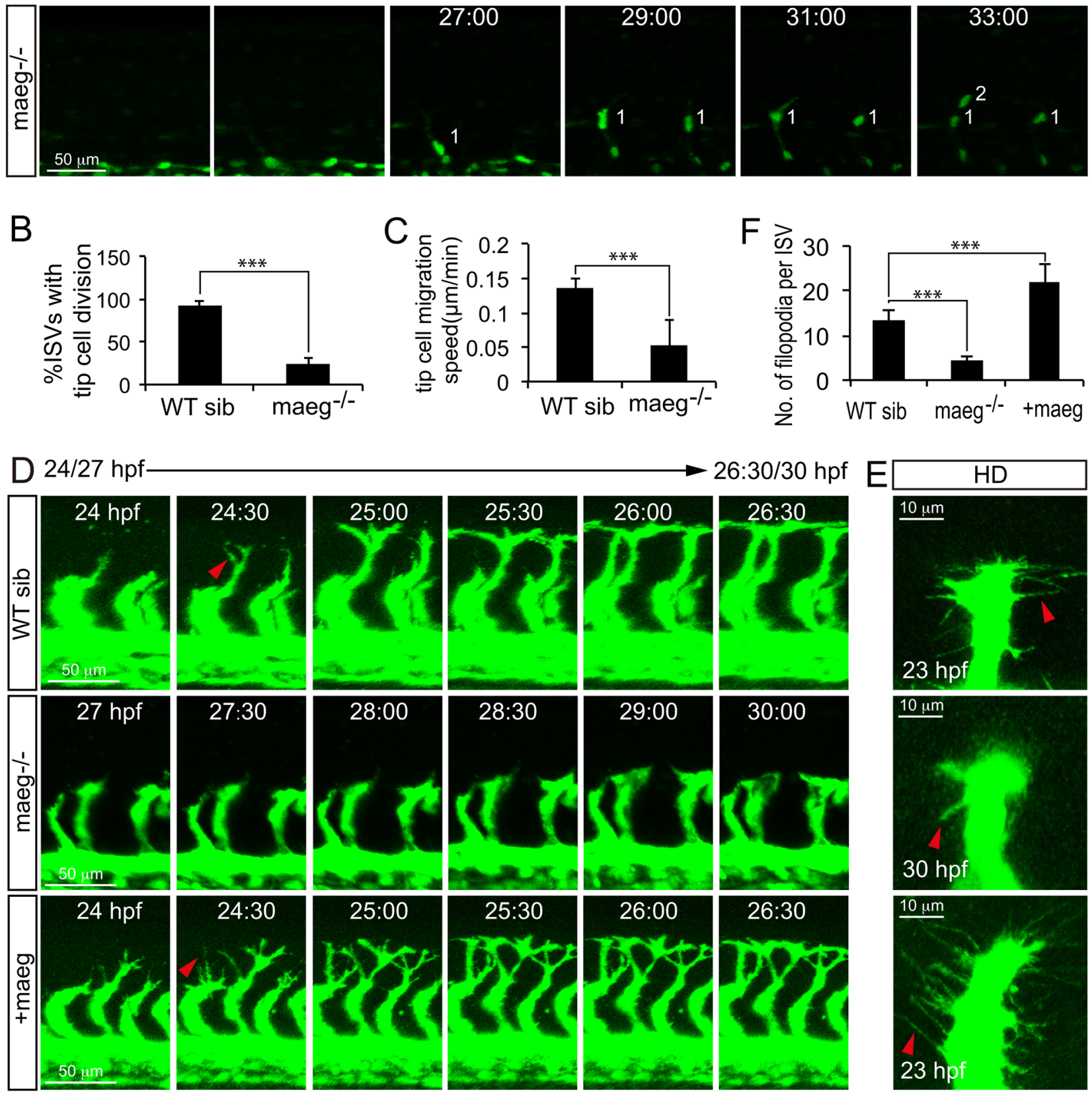

Figure 5: Maeg regulates ISV tip cell behaviors. A. Still images from in vivo time-lapse imaging analysis of WT and maeg $^{-1-} \operatorname{Tg}$ (flila:nEGFP) embryos. Time (hpf) is noted in the top. Nuclei of ISVs are numbered. B. Percentage of ISVs with tip cell division in control embryos and $\mathrm{maeg}^{-/-}$embryos. Student's t-test; ***, $P<0.001$. C. Migration speed of ISV tip cells. Mann Whitney U-test; $* * *, P<0.001$. D. Still images from in vivo time-lapse imaging analysis of ISV tip cell filopodia in $\operatorname{Tg}(k d r l: E G F P)$ embryos. Time (hpf) is noted in the bottom. Red arrowheads indicate filopodia extensions. E. Confocal imaging analysis of ISV tip cell filopodia in $T g(k d r l: E G F P)$ embryos with HD detection setting. Red arrowheads indicate filopodia extensions. F. ISV tip cell filopodia number in per ISVs. One-Way ANOVA; ***, $P<0.001$. 
(Figure 5B). These observations suggest that maeg regulates both migration and proliferation of tip cells during ISV branching angiogenesis. Endothelial tip cells extend dynamic filopodia to sense the surroundings and lead the outgrowth of capillaries. Tip cell filopodia extensions of ISVs appeared shorter and less in maeg mutants (Figure 5D-5F) compared with that of controls (Figure 5D-5F). In contrast, endothelial tip cell filopodia extensions of vessel sprouts appeared more abundant in maeg gain of function embryos (Figure 5D-5F). In addition, the maeg gain of function embryos showed the ectopic branching angiogenic behaviors in the ISV and DLAV (Supplementary Figure S5). These results are in agreement with the branching angiogenic defects in maeg loss and gain of function embryos.

\section{Maeg promotes angiogenesis dependent on RGD domain and mediates activation of Akt/ERK signaling in vivo}

Maeg was reported to contain a RGD domain that binds to its receptor integrins [18, 24]. Through bioinformatical analysis we proved zebrafish Maeg contain a conserved RGD domain as well [22]. To determine whether Maeg regulates angiogenesis through interaction with integrins, we examined the function of RGD domain on the vascular phenotype of maegdeficency zebrafish embryos. Coinjection of an ATGMO-1 resistant form of maeg mRNA significantly reduced the ratio of ISV branching defect (Figure 6B-6iii, 6C). However coinjection of an ATG-MO-1 resistant form of
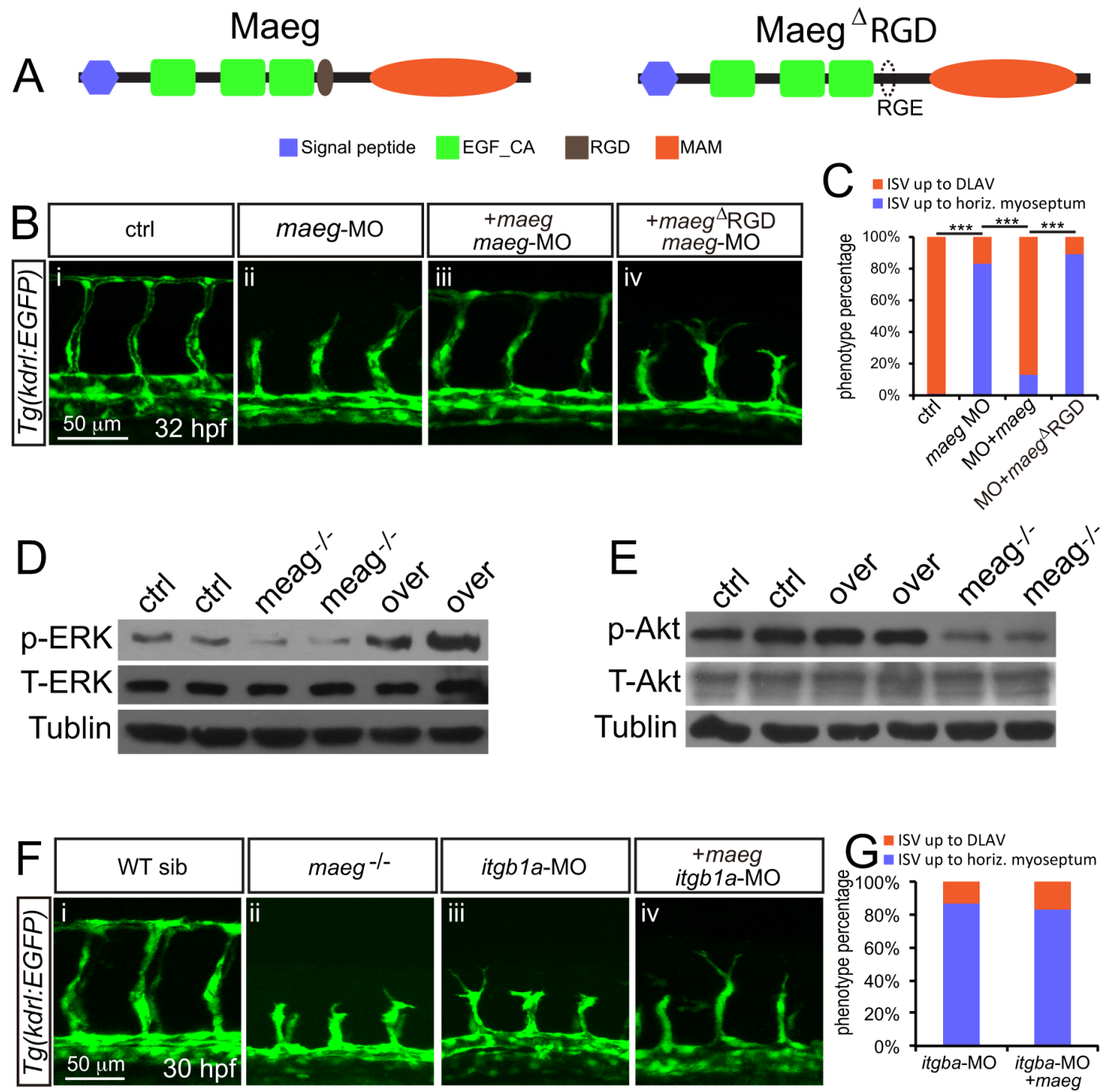

Figure 6: Maeg regulates angiogenesis dependent on RGD domain. A. The diagram of Maeg protein with RGD domain and Maeg protein with RGD domain mutated to RGE domain (Maeg ${ }^{A R G D}$ ). B. Confocal images of ISVs in control embryos, maeg morphants, maeg morphants treated with DAPT, and maeg morphants coinjected with dll4 $\mathrm{MO}$ using $T g(k d r l: E G F P)$ transgenic embryos. C. Percentage of embryos with ISV defect in each group. $\chi^{2}$ test; ***, $P<0.001$. D, E. Phosphorylation levels of ERK and Akt determined by Western blot analysis in maeg loss- and gain-of-function embryos. F. Confocal images of ISVs in control embryos, maeg mutants, itgbla morphants, and itgbla morphants coinjected with maeg mRNA using $T g(k d r l: E G F P)$ transgenic embryos. G. Percentage of embryos with ISV defect in each group. 
maeg mRNA with RDG domain mutated to RGE failed to rescue the ISV length (Figure 6A, B-iv, 6C). These results suggest the maeg regulates embryonic angiogenesis dependent on RGD domain through integrins. Ligation of integrins triggers a large variety of signal transduction events including PI3K/Akt and MEK/ERK [25]. The activation of ERK and Akt pathway is necessary for several key EC functions, including proliferation, migration, survival, and vascular tone [26, 27]. In addition, ERK pathway is strongly activated by Maeg in SVEC (a simian virus 40-transformed mouse microvascular endothelial cell line) [20]. Therefore we examined the effect of Maeg on the activation of ERK and Akt pathways in vivo by Western Blot using specific phosphorylated antibodies to p-ERK and p-Akt. It was shown that the p-ERK and p-Akt was down regulated in maeg mutants (Figure 6D, 6E). Subsequently, we observed that blocking the function of MEK with specific inhibitor U0126 or PD98059 treatment resulted in sprouting angiogenesis defects, as previous reported [28], with reduced length of ISV and decreased number of ECs (Supplementary Figure S6). These phenotypes are reminiscent of those in maeg deficiency embryos. Taken together, these findings indicate ERK is involved in Maeg mediated angiogenesis. Integrin $\beta 1$ (Itgb1) was reported to be one of the receptor for Maeg recognizing the RGD motif [29]. Then we did a series of experiments to testify weather integrin $\beta 1$ was the receptor through which Maeg regulates angiogenesis in zebrafish embryos. Based on the previous studies, there are four itgbl isoforms in zebrafish, including itgbla, $i \operatorname{tgb} 1 b$, itgb1b.1, and itgb1b.2 [30, 31]. We isolated GFP+ cells from $\mathrm{Tg}(k d r l: E G F P)$ transgenic embryos at $24 \mathrm{hpf}$ by fluorescence-activated cell sorting (FACs) (Supplementary Figure S7A). The purity of these sorted cells was validated by FAC resorting and Taqman PCR analysis of marker genes of the ECs (Supplementary Figure S7B, S7C). The expression levels of itgbl isoforms were determined by RT-PCR Assay (Supplementary Figure S7D), showing itgbla was expressed in ECs of $24 \mathrm{hpf}$ zebrafish embryos (Supplementary Figure S7D). These results were confirmed by whole-mount in situ hybridization data (Supplementary Figure S7E-S7H). Subsequently, we knockdown the itgbla using a translation blocking $\mathrm{MO}$ in $T g(k d r l: E G F P)$ zebrafish embryos and observed that itgbla morphants phenocopied the angiogenic defects of maeg mutants (Figure F6F-iii). In addition, overexpression of maeg in itgbla deficiency embryos did not rescue the phenotype of itgbla morphants (Figure F6F-iv, 6G). Taken together, these data suggested that maeg promotes angiogenesis in zebrafish embryos through Integrin $\beta 1$.

\section{Notch signaling in maeg deficiency embryos}

Egf-like family member has been implicated in the modulation of Notch signaling $[16,32,33]$. The Notch signaling pathway has been well documented to be involved in angiogenic cell behavior in ISVs [34, 35]. Therefor we hypothesized maeg promoted zebrafish embryonic branching angiogenesis involving Notch signaling. Then we examined the expression level of Notch receptors and ligands in maeg deficiency embryos. Our data sets showed that the expression level of the notchl $a$ and notch $1 b$ was elevated (Figure 7A-7viii, -ix). These data suggest that maeg inhibits Notch signaling. If the up-regulation of Notch signaling is responsible for the ISV angiogenic defects in maeg deficiency embryos, reducing Notch signaling level will restore the angiogenic potential. To test this hypothesis, we treated the maeg morphants with the Notch $\gamma$-secretase inhibitor DAPT and knock down dll4 expression by MO injection. We found that inhibition of Notch signaling did not completely rescue the ISV length of maeg deficiency embryos (Figure 7B, 7D). But it restored the endothelial cell number (Figure 7C, 7E). Thus, loss of maeg is associated with the increase of Notch, whereas inhibition of Notch is not sufficient to rescue the vascular branching phenotype in maeg loss-of-function embryos. Furthermore, we did a series of experiments to testify whether the Notch signaling is downstream of Itgb1. It was revealed that notchla and deltaC were increased in itgbla morphants (Supplementary Figure S8A). DAPT treatment and downregulation of dll4 partially rescued the ISV branching defects caused by loss of itgbla (Figure 7F-7H, S8B). These data suggest that Notch signaling acts downstream of Itgb1.

\section{DISCUSSION}

EGF-like proteins have been suggested to play a variety of roles in angiogenesis and endothelial cell behaviors. BTC, a member of the EGF family, induces angiogenesis through activation of mitogen-activated protein kinase (MAPK) and phosphatidylinositol 3'-kinase (PI3K) in human umbilical vein endothelial cells (HUVECs) [6]. In addition, using the mouse Matrigel plug assay BTC was proved to be capable of promoting angiogenesis in vivo[6]. HB-EGF belongs to the EGF superfamily of ligands. HB-EGF-induced HUVEC migration and capillary tube formation were dependent upon activation of PI3K and MAPK signaling pathways but were independent of the endothelial cell behaviors induced by VEGF [7-10]. Most secreted angiogenic signaling molecules are mainly produced by non-endothelial cell types. In contrast, EGFL7 is a unique secreted angiogenic factor because it is almost exclusively expressed by and acts on endothelial cells. It is important for regulating tubulogenesis in zebrafish and for controlling vascular patterning and integrity in mice [11, 17]. Its function in blood vessel development is mediated, at least in part, through modulation of Notch signaling and Akt/ERK activation [11-16, 36]. Currently, maeg was demonstrated to promote angiogenesis dependent on RGD domain and mediates activation of Akt/ERK signaling 

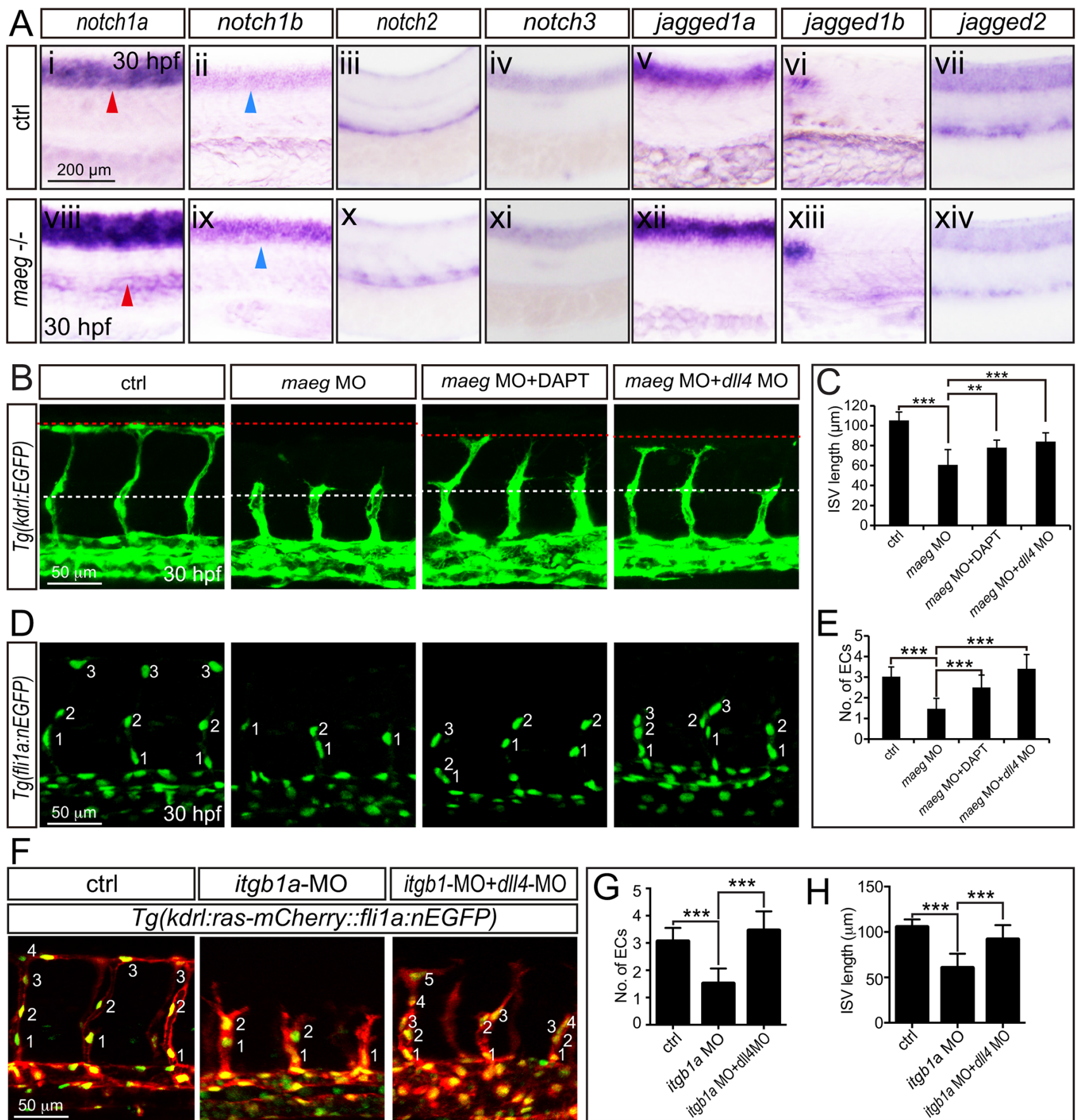

Figure 7: The phenotype of maeg and itgb1 loss-of-function involves Notch signaling. A. Whole mount in situ hybridization analysis of zebrafish embryos using antisense notch $1 a$, notch $1 b$, notch2, notch3, jag1a, jag1b, and jag2b probes. 30 hpf, lateral view. Blue arrowheads indicate the notochord position. Red arrowheads indicate the trunk vessel position. B. Confocal images of ISVs in control embryos, maeg morphants, maeg morphants treated with DAPT, and maeg morphants coinjected with dll4 MO using Tg(kdrl:EGFP) transgenic embryos. The red and white dash lines indicate the position of dorsal roof and horizontal myoseptum respectively. C. The statistics of ISV length in $30 \mathrm{hpf}$ control embryos, maeg morphants, maeg morphants treated with DAPT, and maeg morphants coinjected with dll4 MO. One-Way ANOVA; $* * *, P<0.001$. D. Confocal imaging analysis of endothelial numbers of ISVs in $30 \mathrm{hpf}$ control embryos, maeg morphants, maeg morphants treated with DAPT, and maeg morphants coinjected with dll4 MO using Tg(flila:EGFP) transgenic embryos. Nuclei of ISVs are numbered. E. Quantification of ECs nuclei number in ISV. Measurements were made from three adjacent ISVs (over yolk) per embryo from 3 independent experiments. One-Way ANOVA; $* * *, P<0.001$. F. Confocal images of ISVs in control embryos, itgbla morphants, and itgbla morphants coinjected with dll4 MO using Tg(kdrl:ras-mCherry::flila:nEGFP) transgenic line at 30 hpf. G. Quantification of ECs nuclei number in ISV. Measurements were made from three adjacent ISVs (over yolk) per embryo from 3 independent experiments. One-Way ANOVA; ${ }^{* *}, P<0.001$. H. The statistics of ISV length. One-Way ANOVA; $* * *, P<0.001$. 
in vivo (Figure 8). Zebrafish Maeg and the other several Egf-like proteins share the similar downstream signaling pathway, through which they regulate angiogenesis. In support, Nichol et al showed that 25\% Tie2-Egfl7 transgenic mice exhibited knot-like structure vessels [16]. Similarly, knot-like structures were also observed in ISVs of maeg gain-of-function embryos. The ERK pathway is activated by MAEG whereas Akt remains constant in SEVC (a simian virus 40-transformed mouse microvascular endothelial cell line) cells [20]. This discrepancy is possibly due to the different system was used.

Altered Notch signaling affects several aspects of angiogenesis, including angiogenic cell behavior and tip cell differentiation in segmental sprouts $[34,37]$. Inactivation of maeg in zebrafish embryos impaired ISV out growth and tip cell behaviors that is reminiscent of Notch activation. In
Maeg mutants, Notch signaling was upregulated and this result was consistent with previous studies that Egf-like family members have been implicated in the modulation of Notch signaling. Nueda et al revealed that the EGF-like protein DLK1 Inhibits Notch signaling and potentiates adipogenesis of mesenchymal cells [33]. It was also shown that EGFL7 interacts with receptors of the Notch family and acts as an antagonist of the Notch signaling pathway in cultured neural stem cells [32]. In particular, Nichol et al showed that EGFL7 regulates blood vessel development, at least in part, by modulation of Notch signaling [16]. EGFL7 overexpression in the postnatal retina and in primary endothelial cells reduces Notch target gene expression and induces a subtle hyperangiogenic response, similar to what has been observed when Notch signaling is inhibited [16]. Currently, we provided evidences that inhibition of

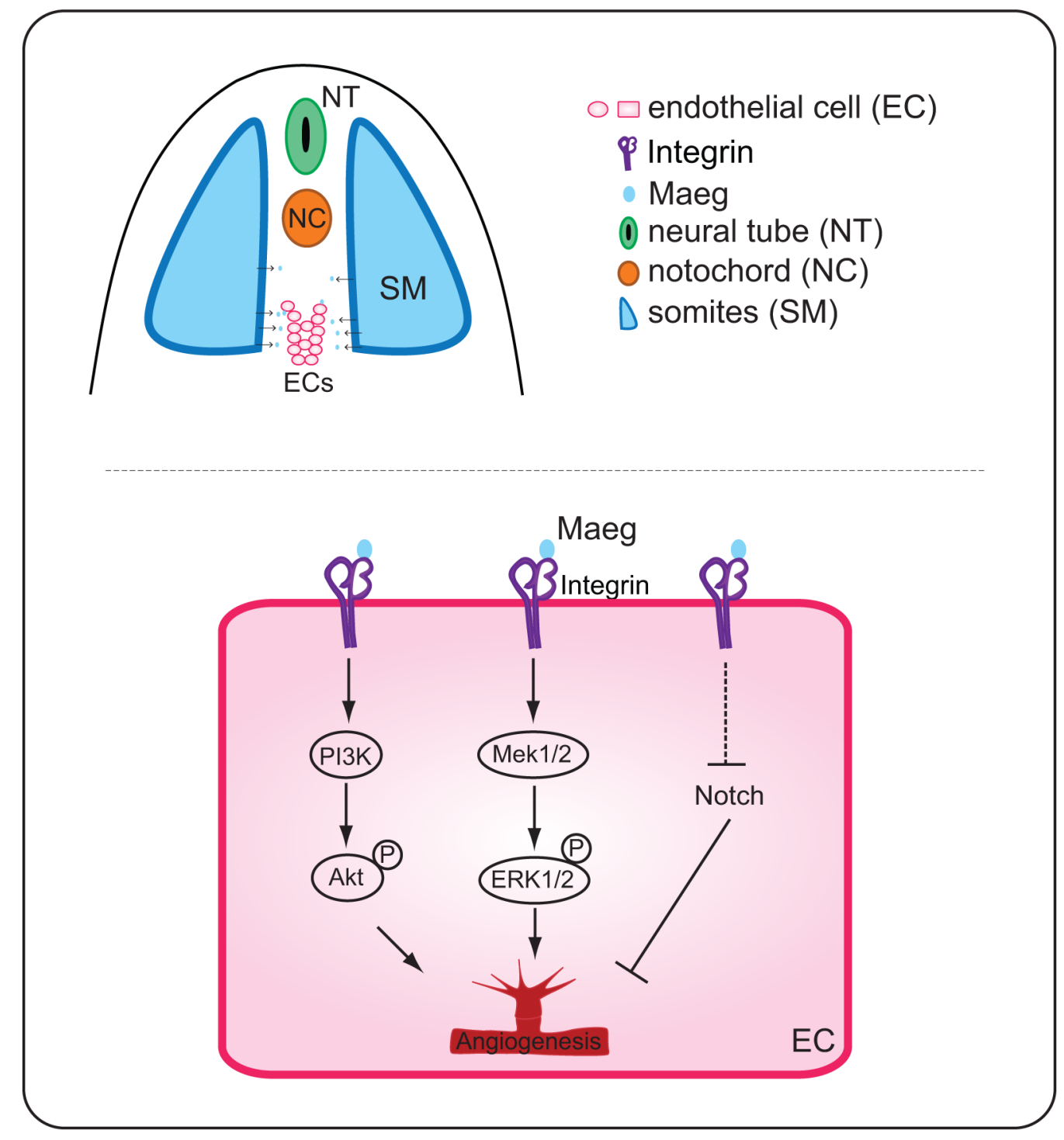

Figure 8: A working model for the function of Maeg in angiogenesis. Binding of Maeg to their receptors on ECs leads to activation of the PI3K/Akt and Mek/ERK signaling pathways, which are involved in angiogenesis. Maeg/Itgb1 negatively regulate Notch signaling, which inhibits angiogenesis. 
Notch signaling partially rescued the angiogenic defects of maeg and itgbl deficiency embryos, suggesting MaegItgb1 modulates angiogenesis upstream of Notch signaling (Figure 8). Taken together, these observations suggested that EGF-like proteins negatively regulate Notch signaling in a number of biological processes including angiogenesis.

We showed that maeg overexpression caused excessive branching of ISVs. In the hyperbranched sprouts, the tip cells project additional filopodia extensions and form connections with sprouts from adjacent ISVs and DLAVs. Most of these connections are lumenized, perfused and are not pruned, suggesting that the aberrant branches develop into functional vessels. Although Maeg overexpression caused hyperangiogenic behaviors of ECs and excessive branching, these embryos did not exactly recapitulate the phenotypes associated with Notch loss-offunction. In dll4 inactivation embryos, the ectopic branches are largely unperfused, whereas the lumen diameter of the branches in maeg gain-of-function embryos is sufficiently large to allow the blood cell perfusion. Furthermore, the locations of the aberrant branches in maeg gain-offunction and dll4 loss-of-function embryos are different. The aberrant branches in dll4 loss-of-function embryos are mostly restricted in the top position of ISV and DLAV, whereas those ectopic branches widely locate in the dorsal half of ISV. Additionally, inhibition of Notch signaling in maeg deficiency embryos partially rescued the angiogenetic defects. These results suggest that maeg promotes angiogenesis via additional signaling pathway more than Notch.

\section{MATERIALS AND METHODS}

\section{Ethics Statement}

All animal experimentation was carried out in accordance with the NIH Guidelines for the care and use of laboratory animals (http://oacu.od.nih.gov/regs/ index.htm) and ethically approved by the Administration Committee of Experimental Animals, Jiangsu Province, China (Approval ID: SYXK (SU) 2007-0021).

\section{Zebrafish strains and breeding}

Zebrafish embryos and adult were raised and maintained under the conditions as we previously described [38-40]. AB and transgenic zebrafish lines: Tg(flila:nEGFP), Tg(kdrl:EGFP), Tg(kdrl:ras-mCherry) and $\operatorname{Tg}(h u C: E G F P)$ were used as described in our previous work [39-42]. Embryos were obtained and treated like we previously did [42].

\section{TALEN construction and microinjection}

We designed a pair of TALENs targeting the first exon of Maeg using online tools TALE-NT (https://tale-nt.cac. cornell.edu/) [43]. Left arm and Right arm of Maeg TALEN use FokI heterodimers. The expression plasmids of the TALENs were constructed and linearized with NotI enzyme. TALEN mRNAs were synthesized in vitro using the linearized constructs as templates with SP6 mMESSAGE mMACHINE Kit (Ambion), purified with RNeasy Mini Kit (Qiagen), and dissolved in RNase free Ultrapure water (Life Technologies). Equal amounts (100 ng/ $\mu \mathrm{l})$ of Left and Right TALEN mRNA were injected together into the cytoplasm of 1-cell stage zebrafish embryos.

\section{TALEN in vivo activity assay and identification of maeg mutants}

The TALEN mRNA injected embryos were maintained in E3 medium $(5 \mathrm{mM} \mathrm{NaCl}, 0.17 \mathrm{mM} \mathrm{KCl}$, $0.33 \mathrm{mM} \mathrm{CaCl} 2,0.33 \mathrm{mM} \mathrm{MgSO} 4)$ at $28.5{ }^{\circ} \mathrm{C} .20$ embryos at $24 \mathrm{hpf}$ after TALEN mRNA injection were collected and their genomic DNA were extracted and subjected to perform high-resolution melting assay (HRMA) [44]. Primers are listed in supplemental data (Supplementary Table S1). The HRM result shows maeg TALEN pair is functional since the melting curve for TALEN mRNA injected embryos is shifted when WT is compared. To identify germ line-transmitted mutations, the microinjected founder (F0) embryos were raised to adulthood. The F0 fish were then outcrossed with wild-type zebrafish to produce F1. 16 of F1 embryos at $24 \mathrm{hpf}$ were collected for genomic DNA extraction respectively. Subsequently, the genomic DNAs were subjected to perform HRMA. Siblings of the F1 embryos that potentially carry heritable mutations were raised to adulthood and individual F1 mutants were identified via PCR amplification and sequencing with fin-clipped DNAs. The primers for genotyping are listed in supplemental data (Supplementary Table S1).

\section{Injection of morpholinos and mRNAs}

Morpholino antisense oligos (MOs; Gene Tools) were prepared at a stock concentration of $1 \mathrm{mM}$ according to the manufacturer's instruction. MOs were diluted to $0.3 \mathrm{mM}$ and injected into one-cell stage embryos. MOs for targeting zebrafish maeg and dll4 were the same as previously described $[22,40]$. The sequence of standard control MO and itgbla (ENSDART00000039700) translation-blocking $\mathrm{MO}$ are listed in supplemental data (Table S1). Zebrafish maeg and $m$ Cherry coding sequence were cloned into PCS2+ vector. The vector template was linearized with NotI Restriction Enzyme (NEB). Sensecapped mRNAs were synthesized with SP6 mMESSAGE mMACHINE Kit (Ambion), purified with RNeasy Mini Kit (Qiagen), and dissolved in RNase free Ultrapure Water (Life Technologies). 2nl maeg and mCherry mixture (1:1) was injected at $100 \mathrm{ng} / \mu \mathrm{l}$ into cytoplasm of $1 / 2$-cell stage zebrafish embryos. 


\section{RNA extraction, reverse transcription, and PCR}

Tissue was homogenized and frozen in TRIzol Reagent (Invitrogen) and stored at $-80^{\circ} \mathrm{C}$. The RNA was extracted following the manufacturer's instruction. $1 \mu \mathrm{g}$ of total RNA was reverse transcribed into cDNA by the use of Transcriptor First Strand cDNA Synthesis Kit (Roche) according to the manufacturer's instructions. Synthesized cDNA was stored at $-20^{\circ} \mathrm{C}$. All PCR amplifications were carried out in a total volume of $50 \mu 1$ using specific primers and Advantage 2 Polymerase Kit (Clontech). The primers for PCR are listed in supplemental data (SupplementaryTable S1).

\section{Whole-mount in situ hybridization, western blot and immunohistochemistry}

Whole-mount in situ hybridization with antisense RNA probes was performed according to the previous description $[38,40]$. The detailed information of these probes and the primers for generating these probes are listed in supplemental data (Table S1) or described in previous work [22, 30, 40]. DIG-labeled RNA sense and antisense probes were made from the linearized plasmids according to the manufacturer's protocol using the DIG RNA Labeling Kit (SP6/T7) (Roche). The polyclonal antibody against zebrafish Maeg was custom designed and made mouse monoclonal to zebrafish Maeg as previous description [22]. The primary antibody to stain somite boundaries was Thbs4b antibody (GTX129646) bought from (GeneTex). P44/42 MAPK (Erk1/2) (137F5) Rabbit mAb (\#4695), Phospho-p44/42 MAPK (Erk1/2) (Thr202/ Tyr204) (197G2) Rabbit mAb (\#4377), and Phospho-Akt (Ser473) (D9E) XP® Rabbit mAb (\#4060) were bought from (Cell Signaling Technology). Rabbit polyclonal AKT Antibody (10176-2-AP) was from (Proteintech Group). The second antibodies used in immunohistochemistry were Goat Anti-Rabbit IgG H\&L (TRITC) (ab6718) from (Abcam) and CF594 Donkey Anti-Rabbit IgG $(\mathrm{H}+\mathrm{L})$ from (Biotium). Monoclonal Anti-Tubulin (Acetylated antibody produced in mouse) was bought from Sigma (T6793). Immunofluorescence staining and western blot was performed as described previously [40, 42].

\section{Drug treatment}

DAPT was purchased from (Sigma-Aldrich) and dissolved in DMSO. DAPT was used at a final concentration of $100 \mu \mathrm{M}$ diluted as our pervious description [40]. U0126 was dissolved according to supplier's information (10 $\mathrm{mM}$ in DMSO). Up to 60 embryos were treated in 12-well plates U0126 (Sigma) diluted from stock in E3 medium with the concentration of $20 \mu \mathrm{M}$. PD98059 MAP Kinase Inhibitor (Selleck Chemicals) was used at final concentration at $20 \mu \mathrm{M}$. E3 medium containing DMSO alone was utilized as control to ensure no effect of the high DMSO treatment.

\section{Histology and microscopy imaging}

Section of the whole-mount in situ-hybridized embryos was performed according to the previous description [38]. The results of in situ hybridization and photos in bright field were documented with an Olympus DP71 camera on an Olympus stereomicroscope MVX10, Leica imaging system on a Leica compound microscope and Zeiss SteREO Discovery V20 microscope with a Zeiss AxioCam HRc camera. For confocal imaging of blood vessel development in zebrafish embryos, they were anesthetized with egg water $/ 0.16 \mathrm{mg} / \mathrm{mL}$ tricaine $/ 1 \% 1$ phenyl-2-thiourea (Sigma) and embedded in $0.6 \%$ low melting agarose. Confocal imaging was performed with a Leica TCS-SP5 LSM. Analysis was performed using Imaris software.

\section{Statistics}

Statistical analysis was performed using GraphPad Prism ${ }^{\circledR}$ version 6.0c. One-Way ANOVA (Dunnett test, Tukey test), Fisher's exact test, Student's t-test, Mann Whitney U-test, and $\chi^{2}$ test were used $(P<0.05)$. When we did the One-Way ANOVA for multiple comparisons test, we chose correction for multiple comparisons.

\section{ACKNOWLEDGMENTS AND FUNDING}

Supported by National Natural Science Foundation of China 81570447, 31201083, 31400918, 81470692.

\section{CONFLICTS OF INTEREST}

The authors declare that they have no conflict of interest.

\section{REFERENCES}

1. Carmeliet P and Jain RK. Angiogenesis in cancer and other diseases. Nature. 2000; 407:249-257.

2. Hanahan D. Signaling vascular morphogenesis and maintenance. Science. 1997; 277:48-50.

3. Carmeliet P. Mechanisms of angiogenesis and arteriogenesis. Nature medicine. 2000; 6:389-395.

4. Buchner G, Orfanelli U, Quaderi N, Bassi MT, Andolfi G, Ballabio A and Franco B. Identification of a new EGFrepeat-containing gene from human $\mathrm{Xp} 22$ : a candidate for developmental disorders. Genomics. 2000; 65:16-23.

5. Appella E, Weber IT and Blasi F. Structure and function of epidermal growth factor-like regions in proteins. FEBS Lett. 1988; 231:1-4.

6. Kim HS, Shin HS, Kwak HJ, Cho CH, Lee CO and Koh GY. Betacellulin induces angiogenesis through activation of mitogen-activated protein kinase and phosphatidylinositol 3'-kinase in endothelial cell. FASEB journal. 2003; 17:318-320. 
7. Mehta VB, Zhou Y, Radulescu A and Besner GE. HB-EGF stimulates eNOS expression and nitric oxide production and promotes eNOS dependent angiogenesis. Growth factors. 2008; 26:301-315.

8. Mehta VB and Besner GE. HB-EGF promotes angiogenesis in endothelial cells via PI3-kinase and MAPK signaling pathways. Growth factors. 2007; 25:253-263.

9. Sato S, Drake AW, Tsuji I and Fan J. A potent anti-HB-EGF monoclonal antibody inhibits cancer cell proliferation and multiple angiogenic activities of HB-EGF. PloS one. 2012; 7:e51964.

10. Ongusaha PP, Kwak JC, Zwible AJ, Macip S, Higashiyama S, Taniguchi N, Fang L and Lee SW. HB-EGF is a potent inducer of tumor growth and angiogenesis. Cancer research. 2004; 64:5283-5290.

11. Parker LH, Schmidt M, Jin SW, Gray AM, Beis D, Pham T, Frantz G, Palmieri S, Hillan K, Stainier DY, De Sauvage FJ and Ye W. The endothelial-cell-derived secreted factor Egfl7 regulates vascular tube formation. Nature. 2004; 428:754-758.

12. Durrans A and Stuhlmann H. A role for Egfl7 during endothelial organization in the embryoid body model system. Journal of angiogenesis research. 2010; 2:4.

13. Nichol D and Stuhlmann H. EGFL7: a unique angiogenic signaling factor in vascular development and disease. Blood. 2012; 119:1345-1352.

14. Nikolic I, Stankovic ND, Bicker F, Meister J, Braun H, Awwad K, Baumgart J, Simon K, Thal SC, Patra C, Harter PN, Plate KH, Engel FB, et al. EGFL7 ligates alphavbeta3 integrin to enhance vessel formation. Blood. 2013; 121:3041-3050.

15. Schmidt M, Paes K, De Maziere A, Smyczek T, Yang S, Gray A, French D, Kasman I, Klumperman J, Rice DS and Ye W. EGFL7 regulates the collective migration of endothelial cells by restricting their spatial distribution. Development. 2007; 134:2913-2923.

16. Nichol D, Shawber C, Fitch MJ, Bambino K, Sharma A, Kitajewski J and Stuhlmann H. Impaired angiogenesis and altered Notch signaling in mice overexpressing endothelial Egfl7. Blood. 2010; 116:6133-6143.

17. Rossi A, Kontarakis Z, Gerri C, Nolte H, Holper S, Kruger $\mathrm{M}$ and Stainier DY. Genetic compensation induced by deleterious mutations but not gene knockdowns. Nature. 2015; 524:230-233.

18. Yeung G, Mulero JJ, Berntsen RP, Loeb DB, Drmanac R and Ford JE. Cloning of a novel epidermal growth factor repeat containing gene EGFL6: expressed in tumor and fetal tissues. Genomics. 1999; 62:304-307.

19. Oberauer R, Rist W, Lenter MC, Hamilton BS and Neubauer H. EGFL6 is increasingly expressed in human obesity and promotes proliferation of adipose tissue-derived stromal vascular cells. Mol Cell Biochem. 2010; 343:257-269.

20. Chim SM, Qin A, Tickner J, Pavlos N, Davey T, Wang H, Guo Y, Zheng MH and Xu J. EGFL6 promotes endothelial cell migration and angiogenesis through the activation of extracellular signal-regulated kinase. J Biol Chem. 2011; 286:22035-22046.

21. Weinstein B. Vascular cell biology in vivo: a new piscine paradigm? Trends in cell biology. 2002; 12:439-445.

22. Wang X, Wang X, Yuan W, Chai R and Liu D. Egfl6 is involved in zebrafish notochord development. Fish physiology and biochemistry. 2015; 41:961-969.

23. Roman BL, Pham VN, Lawson ND, Kulik M, Childs S, Lekven AC, Garrity DM, Moon RT, Fishman MC, Lechleider RJ and Weinstein BM. Disruption of acvrl1 increases endothelial cell number in zebrafish cranial vessels. Development. 2002; 129:3009-3019.

24. Buchner G, Broccoli V, Bulfone A, Orfanelli U, Gattuso C, Ballabio A and Franco B. MAEG, an EGF-repeat containing gene, is a new marker associated with dermatome specification and morphogenesis of its derivatives. Mech Dev. 2000; 98:179-182.

25. Hynes RO. Integrins: bidirectional, allosteric signaling machines. Cell. 2002; 110:673-687.

26. Lee MY, Luciano AK, Ackah E, Rodriguez-Vita J, Bancroft TA, Eichmann A, Simons M, Kyriakides TR, Morales-Ruiz $\mathrm{M}$ and Sessa WC. Endothelial Akt1 mediates angiogenesis by phosphorylating multiple angiogenic substrates. Proceedings of the National Academy of Sciences of the United States of America. 2014.

27. Olsson AK, Dimberg A, Kreuger J and Claesson-Welsh L. VEGF receptor signalling - in control of vascular function. Nature reviews Molecular cell biology. 2006; 7:359-371.

28. Yu PC, Gu SY, Bu JW and Du JL. TRPC1 is essential for in vivo angiogenesis in zebrafish. Circulation research. 2010; 106:1221-1232.

29. Osada A, Kiyozumi D, Tsutsui K, Ono Y, Weber CN, Sugimoto N, Imai T, Okada A and Sekiguchi K. Expression of MAEG, a novel basement membrane protein, in mouse hair follicle morphogenesis. Exp Cell Res. 2005; 303:148-159.

30. Wang X, Li L and Liu D. Expression analysis of integrin beta1 isoforms during zebrafish embryonic development. Gene Expr Patterns. 2014; 16:86-92.

31. Mould AP, McLeish JA, Huxley-Jones J, Goonesinghe AC, Hurlstone AF, Boot-Handford RP and Humphries MJ. Identification of multiple integrin betal homologs in zebrafish (Danio rerio). BMC cell biology. 2006; 7:24.

32. Schmidt MHH, Bicker F, Nikolic I, Meister J, Babuke T, Picuric S, Muller-Esterl W, Plate KH and Dikic I. Epidermal growth factor-like domain 7 (EGFL7) modulates Notch signalling and affects neural stem cell renewal. Nat Cell Biol. 2009; 11:873-U245.

33. Nueda ML, Baladron V, Sanchez-Solana B, Ballesteros MA and Laborda J. The EGF-like protein dlk1 inhibits notch signaling and potentiates adipogenesis of mesenchymal cells. Journal of molecular biology. 2007; 367:1281-1293. 
34. Siekmann AF and Lawson ND. Notch signalling limits angiogenic cell behaviour in developing zebrafish arteries. Nature. 2007; 445:781-784.

35. Hogan BM, Herpers R, Witte M, Helotera H, Alitalo K, Duckers HJ and Schulte-Merker S. Vegfc/Flt4 signalling is suppressed by D114 in developing zebrafish intersegmental arteries. Development. 2009; 136:4001-4009.

36. Takeuchi K, Yanai R, Kumase F, Morizane Y, Suzuki J, Kayama M, Brodowska K, Nakazawa M, Miller JW, Connor KM and Vavvas DG. EGF-like-domain-7 is required for VEGF-induced $\mathrm{Akt} / \mathrm{ERK}$ activation and vascular tube formation in an ex vivo angiogenesis assay. PloS one. 2014; 9:e91849.

37. Jakobsson L, Franco CA, Bentley K, Collins RT, Ponsioen B, Aspalter IM, Rosewell I, Busse M, Thurston G, Medvinsky A, Schulte-Merker S and Gerhardt H. Endothelial cells dynamically compete for the tip cell position during angiogenic sprouting. Nat Cell Biol. 2010; 12:943-953.

38. Huang Y, Wang X, Wang X, Xu M, Liu M and Liu D. Nonmuscle myosin II-B (myh10) expression analysis during zebrafish embryonic development. Gene expression patterns. 2013; 13:265-270.

39. Xu M, Liu D, Dong Z, Wang X, Wang X, Liu Y, Baas PW and Liu M. Kinesin-12 influences axonal growth during zebrafish neural development. Cytoskeleton. 2014; 71:555-563.
40. Krueger J, Liu D, Scholz K, Zimmer A, Shi Y, Klein C, Siekmann A, Schulte-Merker S, Cudmore M, Ahmed A and le Noble F. Flt1 acts as a negative regulator of tip cell formation and branching morphogenesis in the zebrafish embryo. Development. 2011; 138:2111-2120.

41. Jiang Q, Lagos-Quintana M, Liu D, Shi Y, Helker C, Herzog W and le Noble F. miR-30a regulates endothelial tip cell formation and arteriolar branching. Hypertension. 2013; 62:592-598.

42. Wang X, Ling CC, Li L, Qin Y, Qi J, Liu X, You B, Shi Y, Zhang J, Jiang Q, Xu H, Sun C, You Y, Chai R and Liu D. MicroRNA-10a/10b represses a novel target gene mib1 to regulate angiogenesis. Cardiovascular research. 2016; 110:140-150.

43. Doyle EL, Booher NJ, Standage DS, Voytas DF, Brendel VP, Vandyk JK and Bogdanove AJ. TAL EffectorNucleotide Targeter (TALE-NT) 2.0: tools for TAL effector design and target prediction. Nucleic acids research. 2012; 40:W117-122.

44. Dahlem TJ, Hoshijima K, Jurynec MJ, Gunther D, Starker CG, Locke AS, Weis AM, Voytas DF and Grunwald DJ. Simple methods for generating and detecting locus-specific mutations induced with TALENs in the zebrafish genome. PLoS genetics. 2012; 8:e1002861. 